\title{
Do wildlife omnivores and herbivores serve as a source of multidrug-resistant and pathogenic Escherichia coli strains in Poland? Analysis with reference to the host species range
}

\author{
Marcelina Osinska ${ }^{1}$, Aneta Nowakiewicz ${ }^{1}$, Przemysław Zięba ${ }^{2}$, Sebastian Gnat ${ }^{1}$, and \\ Dominik Lagowski ${ }^{1}$ \\ ${ }^{1}$ University of Life Sciences in Lublin \\ ${ }^{2}$ State Veterinary Laboratory in Lublin
}

July 27, 2020

\begin{abstract}
The phenomenon of resistance of Escherichia coli strains in free-living animals has been constantly expanding in recent years. However, the data is still fragmented and, due to the growing threat to public health, there is a constant need to search for and analyse new reservoirs and indicate their role and importance in the circulation of resistance genes. Therefore, the target group in this study were free-living non-predatory animals as reservoirs of drug-resistant and potentially virulent E. coli strains. For isolation, we used a combination of selective media with four antimicrobials. After additional two-stage analysis of strain similarity, we obtained 70 different isolates, including $71.4 \%$ of multidrug-resistant strains. In strains isolated from all species of animals, we determined high resistance to ampicillin (95.7\%), tetracycline (64.3\%), streptomycin (51.4\%), and chloramphenicol $(38.6 \%)$. Every third of the E. coli-positive individual was a carrier of more than one resistant clone. Moreover, four isolates among the resistant strains had the ExPEC pathotype, two had the ETEC pathotype, and another two represented EHEC. Our study confirmed that not only free-living predatory animals are reservoirs of resistance but also many synanthropic species of herbivores and omnivores contribute substantially to the spread of resistant and virulent E. coli strains.
\end{abstract}

Do wildlife omnivores and herbivores serve as a source of multidrug-resistant and pathogenic Escherichia coli strains in Poland? Analysis with reference to the host species range

Marcelina Osińska ${ }^{\mathrm{a}}$, Aneta Nowakiewicz ${ }^{\mathrm{a}}$, Przemysław Zięba ${ }^{\mathrm{b}}$, Sebastian Gnat $^{\mathrm{a}}$, Dominik Lagowski $^{\mathrm{a}}$

${ }^{a}$ University of Life Sciences, Faculty of Veterinary Medicine, Institute of Preclinical Veterinary Sciences, Sub-Department of Veterinary Microbiology, Akademicka 12, 20-033 Lublin, Poland.

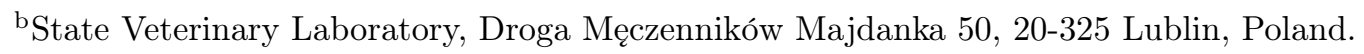

corresponding author : Aneta Nowakiewicz (PhD, DVM),

mail: anowakiewicz@gmail.com or: aneta.nowakiewicz@up.lublin.pl

tel: \#48 814456008

Marcelina Osińska: marcelina.osinska@up.lublin.pl

Przemysław Zięba: przemekzieba@gmail.com

Sebastian Gnat: sebastian.gnat@up.lublin.pl

Dominik Lagowski:dlagowsky@gmail.com 


\section{Summary}

The phenomenon of resistance of Escherichia coli strains in free-living animals has been constantly expanding in recent years. However, the data is still fragmented and, due to the growing threat to public health, there is a constant need to search for and analyse new reservoirs and indicate their role and importance in the circulation of resistance genes. Therefore, the target group in this study were free-living non-predatory animals as reservoirs of drug-resistant and potentially virulent E. coli strains. For isolation, we used a combination of selective media with four antimicrobials. After additional two-stage analysis of strain similarity, we obtained 70 different isolates, including $71.4 \%$ of multidrug-resistant strains. In strains isolated from all species of animals, we determined high resistance to ampicillin (95.7\%), tetracycline $(64.3 \%)$, streptomycin (51.4\%), and chloramphenicol (38.6\%). Every third of the E. coli -positive individual was a carrier of more than one resistant clone. Moreover, four isolates among the resistant strains had the ExPEC pathotype, two had the ETEC pathotype, and another two represented EHEC. Our study confirmed that not only free-living predatory animals are reservoirs of resistance but also many synanthropic species of herbivores and omnivores contribute substantially to the spread of resistant and virulent E. coli strains.

Keywords : ADSRRS fingerprinting, antimicrobial resistance,Escherichia coli, wildlife omnivores and herbivores, virulence-associated genes

\section{Introduction}

Both humans and animals are colonized by microorganisms from the first moments of their lives. Escherichia coli bacteria colonize mainly the large intestine and can enter the organism with food or water and through contact with the surrounding natural world (Katouli, 2010). Most E. coli strains are a natural component of the host microbiota and exist as commensal bacteria; however, there are pathogenic strains that can cause intestinal and extraintestinal infections (Van Elsas, Semenov, Costa, \& Trevors, 2011).

A serious threat to human health posed by E. coli is the capability of easy transfer of resistance and virulence genes between strains. This bacterium has been recognized as an indicator microorganism by the European Centre for Disease Prevention and Control (ECDC) and is used to monitor the occurrence of specific resistance determinants in various sources. For example, according to the ECDC report, the number of $E$. coli strains resistant to the extended spectrum of $\beta$-lactam antibiotics (ESBL) and fluoroquinolones exhibits a growing trend in Poland as well (ECDC, 2019). Moreover, there are many reports of carriage and transmission of multidrug-resistant $E$. coli strains between different host species both in our country and in the world (Nowakiewicz et al., 2020; Hassell et al., 2019; Osińska et al., 2020).

Clinical symptoms caused by $E$. coli are closely correlated with their rich and diverse panel of virulence factors (Chapman et al., 2006). Pathogenic E. coli strains are highly specialized and adapted microorganisms that have developed many features that facilitate pathogenesis, e.g. adhesins, iron acquisition systems, serum resistance, invasins, and miscellaneous factors (Nowak et al., 2017). These strains are generally divided into diarrhoeagenic (IPEC) and extraintestinal (ExPEC) strains. Strains with the ETEC (enterotoxigenic) and EHEC (enterohaemorrhagic) pathotype are mainly responsible for diarrhoea (Dubreuil, Isaacson, \& Schifferli, 2016; Nagy \& Fekete, 2005). However, E. coli strains with the ExPEC pathotype are considered the most common gram-negative pathogens in humans (Wasiński, 2019). ExPEC strains cause many infections in both humans (Khairy, Mohamed, Ghany, \& Abdelrahim, 2019; Dadi et al., 2020) and animals (Nowak et al., 2017; Velhner, Suvajdžić, Todorović, Milanov, \& Kozoderović, 2018; Borges et al., 2017). Unlike E. coli intestinal pathotypes, ExPEC can cause urinary tract infections, pyelonephritis, pneumonia, meningitis, and septicaemia in specific groups in the population such as immunocompromised patients, newborns, and elderly people (Wasiński, 2019). Blood infections caused by ExPEC strains are one of the main causes of hospitalization of patients from nursing homes. These infections are particularly difficult to treat when the strains are resistant to antimicrobials (Bélanger et al., 2011). Moreover, avian pathogenic E. coli (APEC) found in poultry can cause serious losses in the poultry industry. APEC strains attack the respiratory tract of poultry, which further leads to the development of sepsis and generalized infection (Bélanger et al., 2011; Wasiński, 2019). 
Studies conducted over many years have shown the importance of monitoring not only livestock and humans but also different ecosystems and different reservoirs in terms of both resistance and the presence of pathogens including specific pathotypes of E. coli (Dolejska \& Literak, 2019). There are many ways of transferring bacterial strains carrying resistance and virulence genes between wildlife animals, environment, livestock, and humans. Due to the limitation of their territory by urbanization, many species of free-living animals undergo synanthropization, increasingly becoming part of urban and suburban forests and parks. Lower numbers of natural enemies in the environment (reduced population of predators) are also conducive to the increase in the population of large herbivores. Thus, the risk of exchange and spread in both directions (man-wildlife, including the environment) of adverse microorganisms is constantly increasing. Especially in the case of rodents, the spread of bacteria in this group of animals is associated with their specific behaviour, e.g. rats living near households for easy access to food.

Therefore, the aim of our research was to assess whether non-predatory wildlife animals can be substantial reservoirs of resistant and pathogenic E. coli strains only as a result of close contact with the human environment. We hypothesized that not only predatory animals are included in the circulatory cycle of strains that potentially threaten public health.

\section{Materials and methods}

The material for our research were rectal swabs from 108 wildlife omnivorous and herbivorous animals collected from 2014 to 2018 in eastern Poland $(51.216667,22.9)$. Rectal swabs were taken from the following species of animals: common squirrels Sciurus vulgaris $(\mathrm{n}=30)$, rats Rattus $(\mathrm{n}=16)$, European hedgehogsErinaceus europaeus $(\mathrm{n}=8)$, European roe deer Capreolus capreolus $(\mathrm{n}=6)$, red deer Cervus elaphus elaphus $(\mathrm{n}=34)$, European hares Lepus europaeus $(\mathrm{n}=7)$, voles Apodemus agrarius $(\mathrm{n}=2)$, European hamsters Cricetus cricetus $(\mathrm{n}=3)$, and a European beaver Castor fiber $(\mathrm{n}=2)$.

Swabs were collected from animals delivered to the State Veterinary Laboratory in Lublin due to suspected rabies infection, in accordance with the regulations on the control of infectious diseases in animals (Polish Veterinary Inspection, 2015). Poland is still not free from rabies; therefore, suspicious dead animals are tested for the presence of this virus in state laboratories. The sampling procedure was carried out in accordance with the legal recommendations described earlier (Nowakiewicz et al., 2016) and did not require permission of the ethics committee. Only samples with a negative virological result were tested.

\section{Isolation, identification, and selection of strains}

Isolation and identification of resistant $E$. coli strains were carried out according to a procedure described earlier (Osińska et al., 2020). The material was inoculated on selective MacConkey agar media (Biomaxima, Lublin, Poland) supplemented with tetracycline ( $8 \mathrm{mg} / \mathrm{L})$, chloramphenicol (16 mg/L), kanamycin (32 $\mathrm{mg} / \mathrm{L}$ ), and cefotaxime (2 mg/L) (Sigma Aldrich, Germany). Single isolates were selected as described in previous studies (Nowakiewicz et al., 2020; Osińska et al., 2020) using a two-step strain selection approach: the disc diffusion method (DDM) and Amplification of DNA Fragments Surrounding Rare Restriction Sites (ADSRRS).

Based on the analysis of susceptibility profiles consisting of six antimicrobials with DDM, the isolates were considered as different when the susceptibility of the strains differed for at least one drug.

In the second step, based on a similarity value below $90 \%$, the ADSRRS-fingerprinting method was used for distinguishing separate genotypes among strains isolated from the same individual as described in a previous study (Osińska et al., 2020).

DNA was obtained using a ready-made isolation kit (Tissue and Bacterial DNA Purification Kit, Eurx, Gdańsk, Poland).

\section{Determination of phenotypic resistance profiles}

The resistance profiles were determined using the microdilution method. The minimum inhibitory concentration (MIC) was determined for a panel of eleven antimicrobials: ampicillin, cefotaxime, tetracycline, 
chloramphenicol, ciprofloxacin, streptomycin, kanamycin, gentamicin, nalidixic acid, sulfamethoxazole, and nitrofurantoin. The selection of drugs, their concentration, and interpretation of results were carried out in accordance with the CLSI standards (CLSI, 2015), and E. coli ATCC25922 was used as a quality control. A double-disk diffusion test using cefotaxime, cefotaxime/clavulanic acid, ceftazidime, and ceftazidime/clavulanic acid was used to confirm the extended-spectrum $\beta$-lactamase (ESBL) phenotype (CLSI, 2015). Multidrug-resistant strains were defined as being resistant to at least one antimicrobial agent in three or more antimicrobial classes (Magiorakos et al., 2012).

\section{Determination of resistance and virulence gene profiles}

The panel of genes encoding resistance to the following antimicrobials was analysed: tetracycline [tet A, tet B], aminoglycosides [aph (3')-Iaand aph (3' )-IIa], [str A], [aac (3)-II, aac (3)-III], phenicols [cml A, cat, flo R], and sulphonamides [sul 1, sul 2, sul 3]. In order to determine the pathotypes of the strains, the following virulence genes were amplified: genes encoding adhesins $[a f a / d r a B C$, ea-I, papAH, sfa/foc, papC, bfp ], toxins [stx1, stx2, STa, STb, hlyA, eltA], iron acquisition systems [iutA ], serum resistance [kpsMTII ], and miscellaneous factors [esc $V]$.

All amplification reactions were carried out in the thermal cycler (T100 Thermal Cycler, Bio Rad, Hercules, California, USA) using PCR Mix Plus (A\&A Biotechnology, Gdynia, Poland), the GoldTaq mix (Syngen Biotech, Poland), and 1- $\mu$ l DNA sample. The sequence of appropriate primers and reaction conditions (Genomed, Warsaw, Poland) used in this study are described in Table S1.

\section{Statistical analysis}

The relationships of between the percentage of resistant isolates and animal species were identified with one-way ANOVA followed by Bonferroni's post-hoc test; the results were considered statistically significant when the $\mathrm{p}$ value was $<0.05$ (R program, v 3.5.3,https://www.r-project.org/).

\section{Results}

Among all tested rectal swabs, positive growth on the selective MacConkey medium was obtained from 55 animals $(50.9 \%)$. We obtained isolates only from the squirrels, rats, hedgehogs, roe deer, red deer, and beaver (Table S2), and 80 strains were confirmed as E. coli. In total, in the case of 35 individuals, resistant E. colistrains were isolated from only one selective medium (supplemented with one antibiotic: tetracycline, kanamycin, or chloramphenicol). Samples from 15 animals showed growth on two plates with MacConkey medium supplemented with different antibiotics, whereas 15 isolates with positive growth on three plates with antibiotics were recovered from the other five animals (Table S2). Isolates obtained from samples showing growth on two or three different media, we screened using the DDM to eliminate duplicate strains and we reduced the number of strains by another seven isolates (one strain from each animal marked with an asterisk in Table S2). Finally, 73 E. coli isolates were selected for subsequent analyses.

\section{Characterization of genomic profiles with the ADSRRS method}

The isolated strains were characterized by a high degree of diversity of genomic profiles. We found only two pairs of strains at a similarity coefficient of $100 \%$ and one pair with $90 \%$ similarity. The profiles differed in both the number of bands (from 5 to 19) and base pairs of the amplified fragments. Most strains were divided as distinct at a similarity level of $80 \%$ to $15 \%$. We identified 24 different genomic profiles among the squirrels, 14 profiles among the rats, 15 among the red deer, eight profiles for each roe deer and hedgehog, and one for the beaver. We discovered two identical isolates derived from one squirrel sample (No 145): they were isolated simultaneously on the medium supplemented with cefotaxime and chloramphenicol. The other two pairs of strains (J163/TET and J169/TET, J175/CHL and J178/CHL) were isolated from the red-deer samples and each came from a different animal (Fig. S1).

\section{Characterization of multidrug-resistant strains}

Multidrug-resistant strains accounted for $71.4 \%$ of all the different isolated E. coli strains. Ampicillin resistance was observed in $95.7 \%$ of the isolates; however, eight strains were classified as intermediate. Only 
three strains isolated from the rats were found to be susceptible to this antibiotic. More than half of the isolates showed resistance to tetracycline $(64.3 \%)$ or streptomycin $(51.4 \%)$. We also found that $45.7 \%$ of the isolates were resistant to sulfamethoxazole. Kanamycin resistance was observed in 19 strains, and only one strain was resistant to gentamicin (from the squirrel). In total, $38.6 \%$ of the isolates had a chloramphenicol resistant profile, but eight of them were classified as intermediately resistant. Nitrofurantoin resistance was noted in $31.4 \%$ of the strains, but most $(n=16)$ were intermediately resistant isolates. The nalidixic acidresistant isolates accounted for $24.3 \%$, and those with resistance to ciprofloxacin represented $12.9 \%$ (Table S3; Fig. 1).

Among isolates obtained from the squirrels, $70.8 \%$ of the strains $(n=17)$ had a multidrug-resistant profile. In this group of samples, we also detected one E. coli ESBL producer. In addition to resistance to ampicillin and tetracycline, a high proportion (from 37.5 to $45.8 \%$ ) of strains resistant to sulfamethoxazole, chloramphenicol, nalidixic acid, and streptomycin were observed as well.

The multidrug-resistant profile in samples isolated from the rats was observed in $57.1 \%$ of the strains. High aminoglycoside resistance was noted, i.e. $35.7 \%$ for streptomycin and $42.9 \%$ for kanamycin. Almost half of the strains were resistant to nitrofurantoin. Resistance to tetracycline and sulfamethoxazole remained at a high level, as in the case of isolates obtained from the squirrels.

All E. coli strains isolated from the hedgehogs showed a multidrug-resistant profile. In this group, the highest resistance was observed for ampicillin (100\%) and nitrofurantoin (75\%). Moreover, half of the strains were resistant to tetracycline and/or sulfamethoxazole.

The isolates obtained from the ungulates were characterized by high resistance to ampicillin, streptomycin, sulfamethoxazole, and tetracycline. All E. coli strains isolated from roe deer had a multidrug-resistance profile and resistance to five groups of antimicrobials predominated. However, in the case of the red-deer isolates, $53.3 \%(\mathrm{n}=8)$ showed multidrug resistance. Similarly, the analysis of the diversity of resistance depending on the source of the strain (host species) showed statistically significantly higher resistance to tetracycline, streptomycin, sulphametoxazole, and ciprofloxacin among strains isolated from the roe deer. Statistically, resistance to chloramphenicol, kanamycin, nalidixic acid, and nitrofurantoin was most frequently reported in the isolates from the red deer, rats, squirrels, and hedgehog, respectively (Fig 1).

We also examined the single strain obtained from the beaver. It showed a multidrug-resistance profile as well (Table 1).

\section{Molecular characterization of resistance and virulence genes}

We analysed the presence of virulence genes and determinants of resistance to the selected antimicrobials. The str A gene was detected in $75 \%$ of all streptomycin-resistant isolates. Genes conferring kanamycin resistance were only detected in eight out of the 19 resistant strains. The $a p h\left(3^{\prime}\right) I a$ gene was identified in seven isolates, while the $\operatorname{aph}\left(3^{\prime}\right) I I a$ gene was present in only one strain. We detected one strain with resistance to gentamicin encoded by the aac(3)-II gene. The distribution of genes encoding resistance to phenicols was highly diverse. In most chloramphenicol-resistant strains, the flo R gene (16/27) was present alone $(7 / 27)$ or in combination with $\mathrm{cml} \mathrm{A}$ and/or cat . However, 15 strains showed the presence of the $\mathrm{cml}$ A gene, which was found as single (6/27) or with the other analysed genes (Table 2). The tet A gene dominated in the tetracycline-resistant strains and was present as single in 29/45 isolates and together with tet $\mathrm{B}$ in four strains. In ten strains, we found a single $\operatorname{tet} B$ gene. In two cases, we did not detect any of these two genes. Sulfamethoxazole resistance was mainly related to the sul2 gene (26/32) detected as single or together with the other genes tested (Table 2). A single E. coliESBL producer was a carrier of the bla CTX M-27gene.

The pathotypes were determined based on the presence of selected virulence-associated genes (VAGs). We found eight strains with specific pathotypes, and the ExPEC pathotype dominated. We identified theE. coli isolates as ExPEC based on the presence of at least two VAGs including $\mathrm{P}$ fimbrial genes papC, $\mathrm{S}$ fimbrial genessfa/foc, the iron acquisition gene iut $A$, afimbrial adhesion genes afa/dra, and the group 
2 polysaccharide capsule gene kpsMTII, as proposed by Johnson et al. (2003). We found four strains with the ExPEC pathotype. E. coli ExPEC obtained from the squirrels contained the same gene profile: pap C, kpsMTII, iutA, and additionally the papAH gene coding for the pyelonephritis-associated pilus. The R67/KAN strain contained only three genes: pap C, papAH, and $k p s M T I I$, while the H80/CHL strain had additionally the $s f a / f o c$ and $i u t A$ genes and the $\alpha$-hemolysin $h l y A$-coding gene. We also found two isolates from the squirrels that had the ETEC pathotype. The S140/KAN strain had both genes encoding heat-stable enterotoxins, whereas S42/TET had only theestI gene. Two strains, one from the rats (R59/TET) and one from the red deer (J165/TET), were defined as EHEC since they contained the Shigatoxin 2-encoding gene. We did not obtain any EPEC isolate (Table 1).

The comprehensive analysis also showed that 16 animals tested (29\% out of the strain-positive samples) were carriers of more than one E. coli isolate. Moreover, the strains isolated from the same individuals differed in both resistance (phenotypic and gene profiles) and virulence profiles and were characterized by completely separate ADSRRS-fingerprinting profiles (Fig.S1)

\section{Discussion}

In total, we examined 108 rectal swabs originating from a pool of six species of free-living animals and noted positive growth of $E$. coli on the selective MacConkey agar medium in over $50 \%$ of the samples. This is a lower percentage than that in a group of wildlife predators analysed previously, in which we observed resistance to at least one drug in approx. $70 \%$ of the tested animals (Osińska et al., 2020). However, the lower number of resistant isolates in herbivores and omnivores compared to carnivores is consistent with studies in mammals (Nhung et al., 2015) and birds (Smith, Wang, Fanning, \& McMahon, 2014). These differences are related to the type of food consumed by these groups of animals. Carnivores and predators are at the end of the food chain and have more diverse food than herbivores, cumulating all potential resistance determinants from prey. In the case of free-living herbivores, the source of resistant bacteria is mainly assigned to the polluted environment, including plants as a source of food, water, and the ecological niche shared with other animal species (direct and indirect contact).

Using several types of plates supplemented with various antimicrobial substances, we were able to obtain up to 80 bacterial isolates from 55 single animals and, finally, using a two-step method, limit this number to 70 E. coli strains that are distinct in terms of their phenotype, genotype, or origin. This means that every third individual of the resistant $E$. coli -positive animals carries more than one strain, sometimes even three completely separate MDR clones, which may indicate significantly reduced resistance results when analysed according to the strategy "one randomly selected strain isolated from one animal". We also isolated a large number of multidrug-resistant strains, estimated at over $70 \%$, which confirms that regardless the species of host, type of diet, and behaviour, free-living animals are involved in the spread of resistant E. coli strains in Poland despite the lack of direct exposure to antimicrobials (Osińska et al., 2020; Nowakiewicz et al., 2020).

This fact is additionally confirmed by the finding of identical strain clones in two different pairs of the red deer. Unfortunately, we do not have information whether these animals were related to each other or occupied the same area, but this does not change the fact that the same strain was isolated from two different animals even in two cases. Red deer and roe deer live in herds; therefore, close contact may promote a horizontal transfer of resistance genes among animals sharing the same ecological niche (Dolejska \& Literak, 2019). It is also probable that the statistically highest resistance to as many as four different antimicrobials and the multidrug resistance of all the roe deer strains may result from the herd lifestyle (swarm behaviour).

The high resistance to ampicillin, tetracycline, and sulfamethoxazole is also consistent with our previous studies on predatory animals (Osińska et al., 2020). This is also a typical result comparable to those reported in studies of livestock animals in the world (Nhung et al., 2015; de Alcântara Rodrigues, Ferrari, Panzenhagen, Mano, \& Conte-Junior, 2020) and in the same wildlife animal or species groups: ungulates (Wasyl et al., 2018), squirrels (Jalal et al., 2019), and rats (Nkogwe, Raletobana, Stewart-Johnson, Suepaul, \& Adesiyun, 2011; Himsworth et al., 2016).

We also recorded a high level of strain resistance to streptomycin and chloramphenicol, almost identical to 
that in other wildlife animals in Poland (Nowakiewicz et al., 2020; Osińska et al., 2020). As in the previous study, we found only one gentamycin-resistant strain, which confirms that resistance to gentamicin is rather sporadic among $E$. coli isolates from wild animals.

In contrast to our previous studies, lower kanamycin resistance was detected despite the use of this antibiotic for selective isolation of the strains.

The phenotypic resistance of the strains was reflected in most cases in the presence of at least one gene determining resistance to a given antimicrobial. As with E. coli strains isolated from other wildlife or domestic animals and strains isolated from nosocomial infections, the gene profile was very similar, with the predominance of the tet $A$ (tetracycline-resistant strains) and sul 2 or bothsul 1 and sul 2 (sulfamethoxazoleresistant strains) (de Alcântara Rodrigues, Ferrari, Panzenhagen, Mano, \& Conte-Junior, 2020; Hassan et al., 2020).

The nearly $40 \%$ level of chloramphenicol resistance was slightly higher than that recorded previously in wildlife animal studies in Poland (Osińska et al., 2020; Nowakiewicz et al., 2020) and as many as $59 \%$ of the chloramphenicol-resistant strains exhibited the presence of theflo $\mathrm{R}$ gene responsible for both chloramphenicol and florfenicol resistance. The latter antimicrobial is authorized for use in animals also in Poland; therefore, such a high level of resistance to chloramphenicol, although the drug is not approved for use in animals, is likely to be associated with cross-resistance following exposure of animals to florfenicol, residues of which were detected in environmental samples, mainly in water (Hanna et al., 2018). Moreover, florfenicol was considered as persistent against some water-treatment processes (Charuaud et al., 2019). The ESBL phenotype strain had thebla CTX-M -27 gene. This variant has become one of the most common contributors to the spread of $b l a_{\text {CTX-M }}$ in humans in recent years (Cormier et al., 2019). Currently, the producer of the MDR, CTX-M27, ST $131 \mathrm{C} 1$ cluster is considered as a new epidemic clone (Fernandes et al., 2020). Although we did not analyse the strains with the MLST method, it is worth noting that the only strain with the ESBL phenotype isolated from the squirrel was also resistant to the largest number of drug groups (up to seven) among all strains tested similarly as the aforementioned cluster.

Virulence factors are an inherent attribute of pathogenic E. colistrains, unlike commensal strains, which naturally colonize the digestive tract of humans and animals without causing clinical symptoms. As many as $11.4 \%$ of the 70 strains with resistance to at least one antimicrobial exhibited the presence of specific virulence factors, qualifying them to specific pathotypes. However, the percentage of simultaneously pathogenic and drug-resistant strains seems to be lower than in other groups of free-living animals or other food-producing animals from which strains were isolated randomly and not selected for resistance (Nowak et al., 2017; Sarowska et al., 2019). Thus, a high level of resistance is not necessarily accompanied by increased virulence as in our study. We found mainly strains with the ExPEC pathotype. These strains were isolated from the squirrels, rat, and hedgehog samples. Due to the diverse and rich array of virulence factors, it is difficult to determine clearly the origin of ExPEC causing infections in humans. However, all the ExPEC strains in our study were characterized by the presence of genes encoding fimbrial adhesins ( $\mathrm{P}, \mathrm{S}$, and F1C fimbriae) involved in adhesion to epithelial cells in the intestine, kidney, bladder, and lower urinary tract (Kuhnert, Boerlin, \& Frey, 2000; Sarowska et al., 2019) as well as the synthesis of capsular polysaccharide with antiphagocytic activity and ferric aerobactin receptor involved in iron uptake transport (Chapman et al., 2006; Kuhnert, Boerlin, \& Frey, 2000). All these factors were most often detected in UPEC or NMEC strains causing human infections (Sarowska et al., 2019). However, a similar panel of virulence factors was shown in strains from livestock (Bélanger et al., 2011; Wasiński, 2019) and ExPEC strains were isolated from pigs and cattle, where they were responsible for urinary tract infections (Chapman et al., 2006), pneumonia, mastitis, and meningitis (Tan et al., 2011). This pathotype was also found in faecal microbiota of cats and dogs. It was shown that dogs and cats in the same household can be carriers of the same UPEC strains (Johnson, Miller, Johnston, Clabots, \& DebRoy, 2009). This indicates that the strains can overcome the species barrier and spread between animals and humans (Bélanger et al., 2011) (Johnson et al., 2008) (Johnson, Miller, Johnston, Clabots, \& DebRoy, 2009).

Among other wild animal species, wild birds are the most widely studied group in terms of virulence (Borges 
et al., 2017). Borges et al. (2017) discovered the same virulence genes characteristic of APEC pathotype strains that were also found in poultry, wild mammals (Chapman et al., 2006), and humans (Maluta et al., 2014). This phenomenon suggests that wild birds may also transfer virulence genes to ExPEC strains in other hosts (Velhner, Suvajdžić, Todorović, Milanov, \& Kozoderović, 2018) therefore, the virulence factors do not appear to be in any way correlated with the specific source of the strain.

We also found two isolates with the ETEC pathotype among samples collected from the squirrels. Enterotoxigenic E. coli strains are commonly isolated from farm animals and are responsible for diarrhoea, especially in young animals. Since ETEC strains have a narrow host species range due to specific adhesins, they are regarded as strains posing a lower risk to public health. Moreover, enterotoxins are encoded on plasmids, and ETEC strains are characterized by a large genotypic diversity making it difficult to determine their original source (Nagy \& Fekete, 2005). In addition to specific adhesins, ETEC strains can produce two main classes of toxins: heat-stabile (ST) and heat-labile (LT) (Dubreuil, Isaacson, \& Schifferli, 2016). Our strains contained one or two genes responsible for producing ST enterotoxins that are characteristic for ETEC of human, porcine, and calf hosts. Among livestock, ETECs are often isolated from pigs. The pig environment is one of the transmission pathways for ETEC strains. It has been confirmed that ETECs can persist for at least six months when they are protected by manure (Dubreuil, Isaacson, \& Schifferli, 2016). In cattle, typical ETEC strains only produce the STa toxin (Nagy \& Fekete, 2005). This is similar in dogs, but a small proportion of the strains were found to produce the STb toxin as well (Dubreuil, Isaacson, \& Schifferli, 2016). The ETEC pathotype is very rarely isolated from wildlife animals and, due to the low percentage of isolation and insufficient research, the role of these strains in the aetiology of diarrhoea in this group of animals is unknown (Milton et al., 2019).

We also revealed two E. coli strains with the EHEC pathotype present in the squirrel and red deer samples; both of them produced Shigatoxin 2. Shiga-toxin-producing strains (STEC) are a large class of E. coli pathogenic strains and are commonly found in farm and wild animals as well as humans. In humans, infection is mainly caused by the consumption of bacterium-contaminated food or contact with contaminated faeces (Milton et al., 2019). Cattle are the main reservoir of EHEC strains in animals. Unlike humans, EHECs found in adult cattle do not cause disease symptoms but may trigger life-threatening conditions in young animals (Rice, Sheng, Wynia, \& Hovde, 2003). In our research, we only detected the presence of the stx2 gene, but thestx1 gene or both are detected equally often in animal-origin strains (Milton et al., 2019). Stx1 or both genes dominated in cattle (Tavakoli \& Pourtaghi, 2017). Among wild animals, thestx2 gene is detected more frequently in birds (Sanches et al., 2017) and ungulates (Milton et al., 2019). In contrast, in iguanas, strains producing Shiga-toxins accounted for approx. $40 \%$ of pathotypes (Bautista-Trujillo et al., 2020).

\section{Conclusion}

Our hypothesis that not only free-living predatory animals are involved in the spread of resistance has definitely been confirmed by the results of this research. Thus, the diet, the lifestyle associated with living close to human settlements, and even the potential of using the same food sources (rats) probably have a large impact on the emergence of a high percentage of resistance among strains isolated from herbivores, insectivores, or omnivores. Moreover, the large diversity of the studied isolates and the possibility of carrying more than one MDR strain indicate that the results obtained so far showing free-living animals as reservoirs of drug resistance may be significantly underestimated, which seems to be an alarming phenomenon. Furthermore, these MDR strains are carriers of resistance genes, which are also commonly found in strains from humans, farm animals, and pets.

We must also be aware that although $E$. coli is mainly a commensal bacterium, some strains can cause infections. Our research indicates that not only farm animals are a common reservoir for the ExPEC, ETEC, and EHEC strains, which is a serious premise for the need to study not only the spread of resistance genes but also virulence-associated genes in E. coli strains isolated from many species of wild animals.

Funding sources : This research did not receive any specific grant from funding agencies in the public, 
commercial, or not-for-profit sectors.

Ethic statement: The authors confirm that the ethical policies of the journal, as noted on the journal's author guidelines page, have been adhered to. No ethical approval was required

\section{Author Disclosure Statement}

Thereby all authors of the manuscript (Marcelina Osińska, Aneta Nowakiewicz, Przemysław Zieba, Sebastian Gnat and Dominik Eagowski) declare that there is no conflict of interest actual and potential, including any financial, personal, or other relationships with other people or organizations.

\section{Data Availability Statement}

The data that support the findings of this study are available from the corresponding author upon reasonable request

\section{References}

Bautista-Trujillo, G. U., Gutiérrez-Miceli, F. A., Mandujano-García, L., Oliva-Llaven, M. A., IbarraMartínez, C., Mendoza-Nazar, P., ... Gutiérrez-Jiménez, J. (2020). Captive Green Iguana Carries DiarrheagenicEscherichia coli Pathotypes. Frontiers in Veterinary Science, 7(February), 1-9. https://doi.org/10.3389/fvets.2020.00099

Bélanger, L., Garenaux, A., Harel, J., Boulianne, M., Nadeau, E., \& Dozois, C. M. (2011). Escherichia colifrom animal reservoirs as a potential source of human extraintestinal pathogenic E. coli.FEMS Immunology and Medical Microbiology, 62(1), 1-10. https://doi.org/10.1111/j.1574-695X.2011.00797.x

Borges, C. A., Beraldo, L. G., Maluta, R. P., Cardozo, M. V., Barboza, K. B., Guastalli, E. A. L., ... Ávila, F. A. (2017). Multidrug-resistant pathogenic Escherichia coli isolated from wild birds in a veterinary hospital. Avian Pathology, 46(1), 76-83. https://doi.org/10.1080/03079457.2016.1209298

CLSI (2015). Clinical and Laboratory Standard. M100-S25 Performance Standards for Antimicrobial Susceptibility Testing, Twenty-Fifth informational supplement.

Chapman, T. A., Wu, X. Y., Barchia, I., Bettelheim, K. A., Driesen, S., Trott, D., .. Chin, J. J. C. (2006). Comparison of virulence gene profiles of Escherichia coli strains isolated from healthy and diarrheic swine. Applied and Environmental Microbiology, 72(7), 4782-4795. https://doi.org/10.1128/AEM.02885-05

Charuaud, L., Jardé, E., Jaffrézic, A., Liotaud, M., Goyat, Q., Mercier, F., \& Le Bot, B. (2019). Veterinary pharmaceutical residues in water resources and tap water in an intensive husbandry area in France. Science of the Total Environment, 664(May), 605-615. https://doi.org/10.1016/j.scitotenv.2019.01.303

Cormier, A., Zhang, P. L. C., Chalmers, G., Weese, J. S., Deckert, A., Mulvey, M., .. Boerlin, P. (2019). Diversity of CTX-M-positiveEscherichia coli recovered from animals in Canada. Veterinary Microbiology, 231(February), 71-75. https://doi.org/10.1016/j.vetmic.2019.02.031

Dadi, B. R., Abebe, T., Zhang, L., Mihret, A., Abebe, W., \& Amogne, W. (2020). Distribution of virulence genes and phylogenetics of uropathogenic Escherichia coli among urinary tract infection patients in Addis Ababa, Ethiopia. BMC Infectious Diseases, 20(1), 1-12. https://doi.org/10.1186/s12879-020-4844-Z

de Alcântara Rodrigues, I., Ferrari, R. G., Panzenhagen, P. H. N., Mano, S. B., \& Conte-Junior, C. A. (2020). Antimicrobial resistance genes in bacteria from animal-based foods. In Advances in Applied Microbiology (1st ed.). https://doi.org/10.1016/bs.aambs.2020.03.001

Dolejska, M., \& Literak, I. (2019). Wildlife is overlooked in the epidemiology of medically important antibiotic-resistant bacteria. Antimicrobial Agents and Chemotherapy, 63(8). https://doi.org/10.1128/AAC.01167-19

Dubreuil, J. D., Isaacson, R. E., \& Schifferli, D. M. (2016). Animal enterotoxigenic Escherichia coli . EcoSal Plus, 7(1), 1-80. https://doi.org/10.1128/ecosalplus.ESP-0006-2016 
ECDC (2019). European Centre for Disease Prevention and Control. Surveillance of antimicrobial resistance in Europe 2018.

Ewers, C., Bethe, A., Semmler, T., Guenther, S., \& Wieler, L. H. (2012). Extended-spectrum $\beta$-lactamaseproducing and AmpC-producingEscherichia coli from livestock and companion animals, and their putative impact on public health: A global perspective. Clinical Microbiology and Infection, 18(7), 646-655. https://doi.org/10.1111/j.1469-0691.2012.03850.x

Fernandes, M. R., Sellera, F. P., Cunha, M. P. V., Lopes, R., Cerdeira, L., \& Lincopan, N. (2020). Emergence of CTX-M-27-producingEscherichia coli of ST131 and clade C1-M27 in an impacted ecosystem with international maritime traffic in South America. The Journal of Antimicrobial Chemotherapy, 75(6), 1647-1649. https://doi.org/10.1093/jac/dkaa069

Hanna, N., Sun, P., Sun, Q., Li, X., Yang, X., Ji, X., .. Stålsby Lundborg, C. (2018). Presence of antibiotic residues in various environmental compartments of Shandong province in eastern China: Its potential for resistance development and ecological and human risk. Environment International, 114(March), 131-142. https://doi.org/10.1016/j.envint.2018.02.003

Hassan, R., Tantawy, M., Gouda, N. A., Elzayat, M. G., Gabra, S., Nabih, A., .. Sayed, A. A. (2020). Genotypic characterization of multiple drug resistant Escherichia coli isolates from a pediatric cancer hospital in Egypt. Scientific Reports, 10(1), 1-10. https://doi.org/10.1038/s41598-020-61159-z

Hassell, J. M., Ward, M. J., Muloi, D., Bettridge, J. M., Robinson, T. P., Kariuki, S., . . Fèvre, E. M. (2019). Clinically relevant antimicrobial resistance at the wildlife-livestock-human interface in Nairobi: an epidemiological study. The Lancet Planetary Health, 3(6), e259-e269. https://doi.org/10.1016/S2542-5196(19)30083$\mathrm{X}$

Himsworth, C. G., Zabek, E., Desruisseau, A., Jane Parmley, E., Reid-Smith, R., Leslie, M., ... Cox, W. (2016). Avian pathogenicity genes and antibiotic resistance in Escherichia coli isolates from wild norway rats (Rattus norvegicus) in British Columbia, Canada. Journal of Wildlife Diseases, 52(2), 418-421. https://doi.org/10.7589/2015-09-238

Jalal, M. S., Islam, M. Z., Dutta, A., Dhar, P. K., Das, A., Hasan, M. M., .. Ahad, A. (2019). Antibiotic resistant zoonotic bacteria in Irrawaddy squirrel (Callosciurus pygerythrus). Veterinary Medicine and Science, 5(2), 260-268. https://doi.org/10.1002/vms3.138

Johnson, J. R., Johnston, B., Clabots, C. R., Kuskowski, M. A., Roberts, E., \& DebRoy, C. (2008). Virulence genotypes and phylogenetic background of Escherichia coli serogroup O6 isolates from humans, dogs, and cats. Journal of Clinical Microbiology, 46(2), 417-422. https://doi.org/10.1128/JCM.00674-07

Johnson, J. R., Miller, S., Johnston, B., Clabots, C., \& DebRoy, C. (2009). Sharing of Escherichia coli sequence type ST131 and other multidrug-resistant and urovirulent E. coli strains among dogs and cats within a household. Journal of Clinical Microbiology, 47(11), 3721-3725. https://doi.org/10.1128/JCM.01581-09

Johnson, J. R., Murray, A. C., Gajewski, A., Sullivan, M., Snippes, P., Kuskowski, M. A., \& Smith, K. E. (2003). Isolation and molecular characterization of nalidixic acid-resistant extraintestinal pathogenicEscherichia coli from retail chicken products. Antimicrobial Agents and Chemotherapy, 47(7), 2161-2168. https://doi.org/10.1128/AAC.47.7.2161-2168.2003

Katouli, M. (2010). Population structure of gut Escherichia coliand its role in development of extra-intestinal infections. Iranian Journal of Microbiology, 2(2), 59-72.

Khairy, R. M., Mohamed, E. S., Ghany, H. M. A., \& Abdelrahim, S. S. (2019). Phylogenic classification and virulence genes profiles of uropathogenic E. coli and diarrhegenic E. coli strains isolated from community acquired infections. PLoS ONE, 14(9), 1-10. https://doi.org/10.1371/journal.pone.0222441

Kuhnert, P., Boerlin, P., \& Frey, J. (2000). Target genes for virulence assessment of Escherichia coli isolates from water, food and the environment. FEMS Microbiology Reviews, 24(1), 107-117. 
https://doi.org/10.1016/S0168-6445(99)00034-0

Magiorakos, A. P., Srinivasan, A., Carey, R. B., Carmeli, Y., Falagas, M. E., Giske, C. G., .. Monnet, D. L. (2012). Multidrug-resistant, extensively drug-resistant and pandrug-resistant bacteria: An international expert proposal for interim standard definitions for acquired resistance. Clinical Microbiology and Infection, 18(3), 268-281. https://doi.org/10.1111/j.1469-0691.2011.03570.x

Maluta, R. P., Logue, C. M., Casas, M. R. T., Meng, T., Guastalli, E. A. L., Rojas, T. C. G., ... Silveira, W. D. Da. (2014). Overlapped sequence types (STs) and serogroups of avian pathogenic (APEC) and human extra-intestinal pathogenic (ExPEC) Escherichia coli isolated in Brazil. PLoS ONE, 9(8). https://doi.org/10.1371/journal.pone.0105016

Milton, A. A. P., Agarwal, R. K., Priya, G. B., Aravind, M., Athira, C. K., Rose, L., ... Kumar, A. (2019). Captive wildlife from India as carriers of shiga toxin-producing, enteropathogenic and enterotoxigenicEscherichia coli. Journal of Veterinary Medical Science, 81(2), 321-327. https://doi.org/10.1292/jvms.180488

Nagy, B., \& Fekete, P. Z. (2005). Enterotoxigenic Escherichia coli in veterinary medicine. International Journal of Medical Microbiology, 295(6-7), 443-454. https://doi.org/10.1016/j.ijmm.2005.07.003

Nhung, N. T., Cuong, N. V., Campbell, J., Hoa, N. T., Bryant, J. E., Truc, V. N. T., .. Carrique-Mas, J. (2015). High levels of antimicrobial resistance among Escherichia coli isolates from livestock farms and synanthropic rats and shrews in the mekong delta of Vietnam. Applied and Environmental Microbiology, 81(3), 812-820. https://doi.org/10.1128/AEM.03366-14

Nkogwe, C., Raletobana, J., Stewart-Johnson, A., Suepaul, S., \& Adesiyun, A. (2011). Frequency of detection of Escherichia coli, Salmonella spp., and Campylobacter spp. in the faeces of wild rats (Rattus spp.) in Trinidad and Tobago. Veterinary Medicine International, 2011. https://doi.org/10.4061/2011/686923

Nowak, K., Fahr, J., Weber, N., Lübke-Becker, A., Semmler, T., Weiss, S., .. Ewers, C. (2017). Highly diverse and antimicrobial susceptible Escherichia coli display a naïve bacterial population in fruit bats from the Republic of Congo. PLoS ONE, 12(7), 1-18. https://doi.org/10.1371/journal.pone.0178146

Nowakiewicz, A., Zieba, P., Gnat, S., Trościańczyk, A., Osińska, M., Łagowski, D., .. Puzio, I. (2020). Bats as a reservoir of resistant Escherichia coli : A methodical view. Can we fully estimate the scale of resistance in the reservoirs of free-living animals? Research in Veterinary Science, 128(September 2019), 49-58. https://doi.org/10.1016/j.rvsc.2019.10.017

Nowakiewicz, A., Zieba, P., Ziółkowska, G., Gnat, S., Muszyńska, M., Tomczuk, K., ... Trościańczyk, A. (2016). Free-living species of carnivorous mammals in Poland: Red fox, beech marten, and raccoon as a potential reservoir of Salmonella, Yersinia, Listeria spp. and coagulase-positive Staphylococcus . PLoS ONE, 11(5), 1-16. https://doi.org/10.1371/journal.pone.0155533

Osińska, M., Nowakiewicz, A., Zieba, P., Gnat, S., Łagowski, D., \& Trościańczyk, A. (2020). Wildlife Carnivorous mammals as a specific mirror of environmental contamination with multidrug-resistantEscherichia coli strains in Poland. Microbial Drug Resistance, 00(00). https://doi.org/10.1089/mdr.2019.0480

Polish Veterinary Inspection (2015). Animal Health-Regulatory Commitee-presentations: Rabies-Poland, 09-10 Sept. Available at http://ec.europa.eu/food/animals/health/regulatory_committee/presentations_en.htm.

Rice, D. H., Sheng, H. Q., Wynia, S. A., \& Hovde, C. J. (2003). Rectoanal mucosal swab culture is more sensitive than fecal culture and distinguishes Escherichia coli O157:H7-colonized cattle and those transiently shedding the same organism. Journal of Clinical Microbiology, 41(11), 4924-4929. https://doi.org/10.1128/JCM.41.11.4924-4929.2003

Sanches, L. A., Gomes, M. da S., Teixeira, R. H. F., Cunha, M. P. V., Oliveira, M. G. X. de, Vieira, M. A. M., .. Knobl, T. (2017). Captive wild birds as reservoirs of enteropathogenic E. coli 
(EPEC) and Shiga-toxin producing E. coli (STEC). Brazilian Journal of Microbiology, 48(4), 760-763. https://doi.org/10.1016/j.bjm.2017.03.003

Sarowska, J., Futoma-Koloch, B., Jama-Kmiecik, A., Frej-Madrzak, M., Ksiazczyk, M., Bugla-Ploskonska, G., \& Choroszy-Krol, I. (2019). Virulence factors, prevalence and potential transmission of extraintestinal pathogenic Escherichia coli isolated from different sources: Recent reports. Gut Pathogens, 11(1), 1-16. https://doi.org/10.1186/s13099-019-0290-0

Smith, S., Wang, J., Fanning, S., \& McMahon, B. J. (2014). Antimicrobial resistant bacteria in wild mammals and birds: A coincidence or cause for concern? Irish Veterinary Journal, 67(1), 2-4. https://doi.org/10.1186/2046-0481-67-8

Tan, C., Xu, Z., Zheng, H., Liu, W., Tang, X., Shou, J., .. Chen, H. (2011). Genome sequence of a porcine extraintestinal pathogenicEscherichia coli strain. Journal of Bacteriology, 193(18), 5038. https://doi.org/10.1128/JB.05551-11

Tavakoli, M., \& Pourtaghi, H. (2017). Molecular detection of virulence genes and multi-drug resistance patterns in Escherichia coli(STEC) in clinical bovine mastitis: Alborz province, Iran. Iranian Journal of Veterinary Research, 18(3), 208-211. https://doi.org/10.22099/ijvr.2017.4224

Van Elsas, J. D., Semenov, A. V., Costa, R., \& Trevors, J. T. (2011). Survival of Escherichia coli in the environment: Fundamental and public health aspects. ISME Journal, 5(2), 173-183. https://doi.org/10.1038/ismej.2010.80

Velhner, M., Suvajdžić, L., Todorović, D., Milanov, D., \& Kozoderović, G. (2018). Avian pathogenic Escherichia coli : diagnosis, virulence and prevention. Archives of Veterinary Medicine, 11(2), 21-31. Retrieved from https://niv.ns.ac.rs/wp-content/uploads/Arhiv/AVM-V11-BR2-3.pdf

Wasiński, B. (2019). Extra-intestinal pathogenic Escherichia coli- threat connected with food-borne infections. Annals of Agricultural and Environmental Medicine, 26(4), 532-537. https://doi.org/10.26444/aaem/111724

Wasyl, D., Zajac, M., Lalak, A., Skaryńska, M., Samcik, I., Kwit, R., .. S Szulowski, K. (2018). Antimicrobial resistance inEscherichia coli isolated from wild animals in Poland. Microbial Drug Resistance, 24(6), 807815. https://doi.org/10.1089/mdr.2017.0148

\section{Legends to Tables and Figures}

Table 1. Characterization of resistant E. coli strains

+ Profiles were created from the first letters of the names of antimicrobials to which given strains are resistant: A- ampicillin, C-ciprofloxacin, N- nalidixic acid, Nf - nitrofurantoin, S- streptomycin, G- gentamycin, Ttetracyclin, Sx- sulfamethoxazole, Ch- chloramphenicol, K-kanamycin

Table 2. Distribution of resistance genes in E. colistrains

+AMP- ampicillin, CHL-chloramphenicol, CIP -ciprofloxacin, GEN- gentamycin, KAN-kanamycin, NALnalidixic acid, NFT - nitrofurantoin, STR-streptomycin, SUL-sulfamethoxazole, TET-tetracycline, CTXcefotaxime

$++\mathrm{N}$ - Number of resistant isolates

Fig. 1 Differences in the percentage of phenotypic resistance depending on the host species

* represent the statistical highest resistance to a given drug among the tested species $(\mathrm{p}<0.05)$

Table S1. Primers used in this study

Table S2. Distribution of all cultured E. coli isolates $(\mathrm{n}=80)$ 
+ Growth on plate with: KAN- resistant to kanamycin, TET- resistant to tetracycline, CHL- resistant to chloramphenicol

${ }^{++}$Symbol and number of positive E.coliresistant animal (one asterisk indicates animals in which two isolates with the same resistance profile have been demonstrated using the DDM method, bold indicate animal in which identical ADSRRS profile have been demonstrated using fingerprinting method)

Table S3. Drug susceptibility of E.coli strains

+ AMP- ampicillin, CHL-chloramphenicol, CIP -ciprofloxacin, GEN- gentamycin, KAN-kanamycin, NALnalidixic acid, NFT - nitrofurantoin, STR-streptomycin, SUL-sulfamethoxazole, TET-tetracycline, CTXcefotaxime

Fig. S1 ADSRRS-fingerprinting profiles of E. colistrains

\section{Hosted file}

Table 1.docx available at https://authorea.com/users/346573/articles/472530-do-wildlifeomnivores-and-herbivores-serve-as-a-source-of-multidrug-resistant-and-pathogenicescherichia-coli-strains-in-poland-analysis-with-reference-to-the-host-species-range

\section{Hosted file}

Table 2.docx available at https://authorea.com/users/346573/articles/472530-do-wildlifeomnivores-and-herbivores-serve-as-a-source-of-multidrug-resistant-and-pathogenic-

escherichia-coli-strains-in-poland-analysis-with-reference-to-the-host-species-range

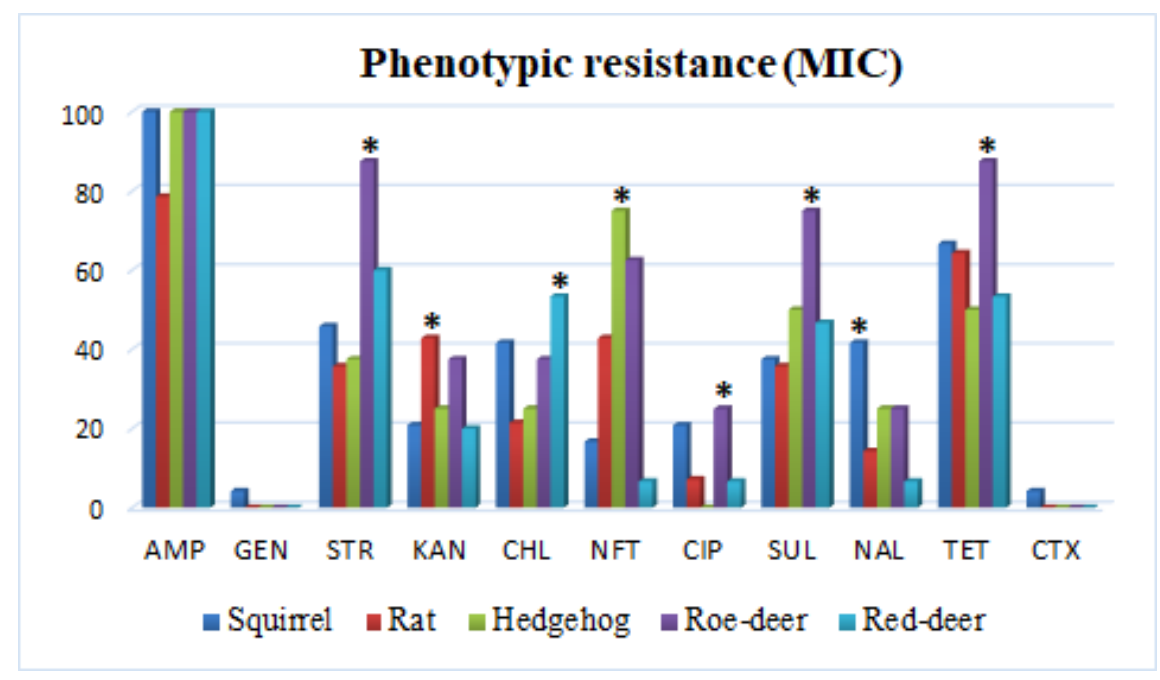

